\title{
HIV Intertest Interval among MSM in King County, Washington
}

\author{
David A. Katz ${ }^{\mathrm{a}}$, Julia C. Dombrowskib,c , Fred Swansond, Susan Buskinn ${ }^{\mathrm{a}, \mathrm{c}}$, Matthew R. \\ Golden $^{\mathrm{a}, \mathrm{b}, \mathrm{c}}$, and Joanne D. Stekler ${ }^{\mathrm{a}, \mathrm{b}, \mathrm{c}}$ \\ aDepartment of Epidemiology, University of Washington, Seattle, WA, US \\ bDepartment of Medicine, University of Washington, Seattle, WA, US \\ cHIV/STD Program, Public Health - Seattle \& King County, Seattle, WA, US \\ dGay City Health Project, Seattle, WA, US
}

\begin{abstract}
OBJECTIVES-We examined temporal trends and correlates of HIV testing frequency among men who have sex with men (MSM) in King County, WA.

METHODS-We evaluated data from MSM testing for HIV at the Public Health - Seattle \& King County (PHSKC) STD Clinic and Gay City Health Project (GCHP) and testing history data from MSM in PHSKC HIV surveillance. The intertest interval (ITI) was defined as the number of days between the last negative HIV test and the current testing visit or first positive test. Correlates of the $\log _{10}$-transformed ITI were determined using GEE linear regression.
\end{abstract}

RESULTS-Between 2003 and 2010, the median ITI among MSM seeking HIV testing at the STD Clinic and GCHP were 215 (IQR: 124-409) and 257 (IQR: 148-503) days, respectively. In multivariate analyses, younger age, having only male partners, and reporting $\geq 10$ male sex partners in the last year were associated with shorter ITIs at both testing sites $(\mathrm{p}<0.05)$. Among GCHP attendees, having a regular healthcare provider, seeking a test as part of a regular schedule, and inhaled nitrite use in the last year were also associated with shorter ITIs $(\mathrm{p}<0.001)$. Compared to MSM testing HIV-negative, MSM newly diagnosed with HIV had longer ITIs at the STD Clinic (median of 213 versus 278 days; $\mathrm{p}=0.01$ ) and GCHP (median 255 versus 359 days; $\mathrm{p}=0.02$ ).

CONCLUSIONS-Although MSM in King County appear to be testing at frequent intervals, further efforts are needed to reduce the time that HIV-infected persons are unaware of their status.

\section{Keywords}

HIV screening; men who have sex with men; testing frequency; late diagnosis

Corresponding author: David A. Katz, MPH, University of Washington, 325 Ninth Avenue, Box 359909, Seattle, WA 98104, Telephone: 206-616-5578, Fax: 206-744-3693, dkatz7@u.washington.edu. Requests for reprints should be addressed to corresponding author.

\section{COMPETING INTERESTS}

None declared.

\section{LICENCE STATEMENT}

The Corresponding Author has the right to grant on behalf of all authors and does grant on behalf of all authors, an exclusive licence (or non exclusive for government employees) on a worldwide basis to the BMJ Publishing Group Ltd to permit this article (if accepted) to be published in Sexually Transmitted Infections and any other BMJPGL products and sublicences such use and exploit all subsidiary rights, as set out in our licence http://group.bmj.com/products/journals/instructions-for-authors/licence-forms. 


\section{BACKGROUND}

HIV testing serves as both the entry point into care for HIV-infected individuals and an effective prevention intervention because behavior change and antiretroviral treatment can reduce ongoing transmission[1,2]. The United States Centers for Disease Control and Prevention (CDC) recommends testing at least annually for individuals at high risk of acquiring HIV, including men who have sex with men (MSM)[3]. More frequent screening (i.e. every 3-6 months) is recommended for MSM who have multiple or anonymous partners, have sex in conjunction with illicit drug use, or have partners who participate in these activities[4]. Similarly, Public Health -Seattle \& King County (PHSKC) recommends annual testing for all sexually-active MSM and quarterly testing for MSM at greater risk of acquiring HIV as identified by locally-derived criteria[5,6].

Despite such recommendations, testing patterns and correlates of HIV testing frequency among MSM are not well understood. Helms et al. described the use of the HIV intertest interval (ITI), the time between the last reported test and the current test among individuals seeking testing, as an estimate of testing frequency[7]. In their analysis of MSM attending STD Clinics in four U.S. metropolitan areas, younger age, black race, and later year of visit were associated with shorter ITIs. Other studies of testing patterns have primarily measured whether individuals have tested in the last year[8-11], although adherence to testing recommendations has also been examined[12].

As a measure of testing frequency, ITIs could be used to monitor the success of HIV testing programs [13], just as community viral load has been proposed as a method for monitoring linkage to care and treatment of HIV-infected persons[14]. We examined temporal trends and correlates of ITIs among MSM in King County, Washington, in order to assess the rate of HIV testing in this population and identify client characteristics to better target HIV testing services and social marketing messages regarding testing.

\section{METHODS}

Data Sources

We examined data from three sources: (1) electronic medical records of MSM testing for HIV at the PHSKC STD Clinic from January 1, 2003 until October 6, 2010; (2) individual level data from MSM testing for HIV at the Gay City Health Project's (GCHP) publiclyfunded HIV/STD testing program targeting MSM from February 2, 2004 until October 6, 2010; and (3) data from PHSKC core HIV/AIDS surveillance and HIV incidence surveillance from January 1, 2004 until October 6, 2010. The initial dates for each data source vary by when complete data was available. The date the STD Clinic converted from clinician-obtained medical and sexual histories to a computer-assisted self-interview data collection system was chosen as the end date for all sources. All men who reported having sex with men in the last year were included as MSM. All MSM diagnosed with HIV in King County are included in PHSKC surveillance data, including those first diagnosed at the STD Clinic and GCHP.

PHSKC defines "high-risk" MSM as those MSM who meet at least one of the following criteria during the past year: 10 or more oral or anal male sex partners; unprotected anal intercourse with a partner of unknown or discordant HIV status; diagnosis of chlamydial infection, gonorrhea, or early syphilis; or use of methamphetamine or inhaled nitrites[5]. Quarterly testing is recommended for these MSM, and annual testing is recommended for all other sexually-active MSM[6]. 


\section{Outcome Ascertainment}

For men attending the STD Clinic or GCHP, the ITI was defined as the number of days between the most recent HIV test and current HIV testing visit. Full dates (day, month, and year) were available from clinic records for the current visit. The date of the most recent test was routinely obtained by self-report during the clinical visit or, for some men who had previously attended the STD Clinic, may have been obtained directly from the medical record. Dates for the most recent test were recorded as month and year or year only; when necessary, days and months were imputed as previously described[7].

In surveillance data, the ITI was defined as the number of days between the most recent HIV-negative test and first HIV-positive test. These dates were obtained by self-report during an interview or by standard medical record reviews conducted by PHSKC staff or medical providers. When self-reported and documented dates differed, the later date was used for the most recent negative test and the earlier date was used for the first positive test.

In all cases, visits at which ITIs were less than 30 days were assumed to be follow-up tests (i.e. not new tests) and were not included in this analysis. In surveillance data, this includes men with acute HIV infection who had their last negative antibody test on the same date as their first positive RNA test. We considered ITIs of 30 to 90 days consistent with quarterly testing and ITIs of fewer than 365 days consistent with at least annual testing.

\section{Data Analysis}

ITIs were $\log _{10}$-transformed in all analyses in order to identify proportional (instead of absolute) increases or decreases in the average ITI per unit change in potential covariates, as described previously[7]. Among MSM testing HIV-negative, temporal trends in ITIs were examined using generalized estimating equations (GEE) linear regression with exchangeable working correlation and robust standard errors to adjust for multiple visits per person. Temporal trends among MSM newly diagnosed with HIV were examined using linear regression. Correlates of ITIs were determined among men attending the STD Clinic and GCHP separately because several variables of interest (education, homelessness, having a regular healthcare provider and health insurance, and testing as part of a regular schedule) were available only for GCHP. GEE models were also used for univariate and multivariate analyses of sociodemographic and HIV risk characteristics. Multivariate analyses included all sociodemographic and risk factors associated with ITIs in univariate analyses $(\mathrm{p}<0.05)$ unless responses were not available for the entire study period. Multivariate models were adjusted for visit year. The association between being newly diagnosed with HIV and ITIs was assessed in separate GEE models, adjusting for age and year of visit. The exponentiated coefficient in these models is the $\mathrm{x}$-fold increase or decrease in the ITI for each one-unit increase in the correlate. Data were analyzed using Stata statistical software version 11.0 (College Station, Texas, U.S.). The study has ethical approval from the Institutional Review Board of the University of Washington.

\section{RESULTS}

Sociodemographic and HIV risk characteristics of the populations studied are described in Table 1. The three populations were similar with respect to age, race, and ethnicity. MSM attending the STD Clinic were more likely to have had sex with both men and women than those attending GCHP (16\% vs. $10 \%)$ and were generally at greater risk for acquiring HIV based on PHSKC risk criteria. At the STD Clinic, 357 (2.5\%) of HIV tests were positive versus $154(1.6 \%)$ of tests at GCHP. 


\section{PHSKC STD Clinic}

Between January 1, 2003 and October 6, 2010, there were 15,326 visits to the STD Clinic by MSM seeking HIV testing who reported a prior negative test or did not know their status. In 14,385 (94\%) of these visits, clients provided dates for their most recent test or reported no prior test. In the remaining visits, the most recent test date was missing $(n=541)$, reported to be after the current visit $(\mathrm{n}=35)$, or within 30 days of the current visit and considered a follow-up test $(\mathrm{n}=365)$.

These 14,385 visits were made by 8029 MSM, who made one to 22 visits during this time period; 2619 (33\%) of 8029 clients were repeat visitors. At these 14,385 visits, 868 (6\%) MSM reported no prior test. At 13,517 visits by men who reported testing previously, ITIs ranged from 30 to 8410 days with a median of 215 days [interquartile range (IQR): 124409; Figure 1]. Men reported an ITI consistent with quarterly testing or with at least annual testing at $13 \%$ and $66 \%$ of all visits, respectively; in $18 \%$ of visits, MSM reported no test in the last two years.

\section{Gay City Health Project}

Between February 2, 2004 and October 6, 2010, there were 10,513 visits to GCHP by MSM seeking HIV testing who reported a prior negative test or did not know their status. In 9851 (94\%) of these visits, clients provided dates for their most recent test or reported no prior test. In the remaining visits, the most recent test date was missing $(n=551)$, reported to be after the current visit $(n=7)$, or considered a follow-up test $(n=104)$.

These 9851 visits were made by 6147 MSM, who made one to 14 visits during this time period; 1796 (29\%) of 6147 clients were repeat visitors. At these 9851 visits, 856 (9\%) MSM reported no prior test. At 8995 visits by men who reported testing previously, ITIs ranged from 30 to 8690 days with a median of 257 days (IQR: 148-503; Figure 1). Men reported an ITI consistent with quarterly testing or with at least annual testing at $8 \%$ and $58 \%$ of all visits, respectively; in $24 \%$ of visits, MSM reported no test in the last two years.

\section{Surveillance}

Between January 1, 2004 and October 6, 2010, 1525 MSM were identified in core HIV/ AIDS and HIV incidence surveillance as newly diagnosed with HIV in King County. For $951(62 \%)$ of these men, dates were available for the first positive and last negative tests, and $81(5 \%)$ men had never tested prior to being diagnosed. For the remainder, it was unknown whether they had a prior negative test $(n=462)$, the last negative and first positive tests were recorded as occurring the same day $(\mathrm{n}=30)$, or the last negative test was recorded as having occurred after the first positive test $(n=2)$.

Of the 1031 MSM with testing histories, 81 (8\%) reported no prior test. Among 950 men who reported testing previously, ITIs ranged from 30 to 8019 days with a median of 365 days (IQR: 183-883; Figure 1). Among all men with testing histories, 13\% and 49\% reported an ITI consistent with quarterly testing or with at least annual testing, respectively; $35 \%$ of newly-diagnosed men reported no test in the last two years.

\section{Temporal Trends in ITIs}

Figure 2 depicts temporal trends in ITIs, stratified by HIV status and population. From 2004 to 2010, the median ITI decreased significantly among MSM testing both newly HIVpositive and -negative at GCHP ( $\mathrm{p}<0.01$ for both). Similarly, at the STD Clinic, the median ITI decreased from 2003 to 2010 among MSM testing both newly HIV-positive and negative, although this change was not significant ( $p>0.05$ for both). Among HIV-positive MSM in surveillance, no changes were observed in ITIs between 2004 and $2010(\mathrm{p}=0.59)$. 


\section{Correlates of ITIs}

Table 2 presents the results of multivariate analyses. All variables significantly associated with ITIs in univariate analyses were included except for history of a bacterial STI, which was not included in the GCHP analysis because it was only available from visits starting in 2008. At the STD Clinic, younger age, sex with men only in the last year, 10 or more male sex partners in the last year, and a history of STI were associated with shorter ITIs ( $p<0.001$ for all). At GCHP, younger age, sex with men only in the last year, 10 or more male sex partners in the last year, inhaled nitrite use in the last year, having a regular healthcare provider, and testing for HIV regularly were associated with shorter ITIs ( $\mathrm{p}<0.05$ for all).

Race, Hispanic ethnicity, being born outside the U.S., methamphetamine use in the last year, injection drug use in the last year, and reporting testing due to concern about potential symptoms of primary HIV infection were not associated with ITIs in either population ( $>0.05$ for all). In addition, among GCHP attendees, education, being homeless, having health insurance, and reporting unprotected anal sex with an HIV-infected male partner or male partner of unknown status in the last year were not associated with ITIs ( $p>0.05$ for all).

\section{HIV Status and ITIs}

At the STD Clinic, the median ITI was 278 days (IQR: 161 to 579) among MSM newly diagnosed with HIV and 213 days (IQR: 123-405) among those testing HIV-negative. Adjusting for age and visit year, newly-diagnosed men had 1.13-fold (95\% CI: $1.03-1.25)$ longer ITIs compared with men testing negative $(\mathrm{p}=0.009)$. At GCHP, the median ITI was 359 days (IQR: 203 to 719) among newly-diagnosed MSM and 255 days (IQR: 147 to 500) among those testing negative. Adjusting for age and visit year, newly-diagnosed men had 1.20-fold (95\% CI: 1.03-1.40) longer ITIs compared with men testing negative ( $\mathrm{p}=0.017)$. At both locations, there were no differences in the proportions of men who reported no prior test between MSM testing negative and those newly diagnosed with HIV (data not shown).

\section{DISCUSSION}

\section{The ITI in King County}

From 2003 to 2010, the median ITI among MSM attending the PHSKC STD Clinic and GCHP were both less than nine months. Over $90 \%$ of men reported prior testing, more than half reported ITIs consistent with at least annual testing, and about one-tenth reported ITIs consistent with quarterly testing. Over time, ITIs declined among men testing both HIVpositive and negative at both clinics, although this change was only statistically significant at GCHP. Encouragingly, these data indicate that many MSM in King County test relatively frequently and that, at least at large publicly-funded testing sites, testing frequency has increased over time. However, among MSM newly diagnosed with HIV, this increase appears to have been driven by changes in the early 2000s and was not replicated in surveillance data. Increases in testing frequency early in the study period may be explained by increased demand for testing following the introduction of rapid testing and pooled nucleic acid amplification testing in late 2003 and recommendations for quarterly testing among high risk MSM in 2005.

\section{Public Health Applications of the ITI}

Insofar as ITIs among individuals newly diagnosed with HIV have the potential to estimate the timing of HIV diagnosis[13], these data suggest that approximately two-thirds of MSM in King County are being diagnosed no more than two years after infection, and only onesixth could have been infected for more than five years. If we estimate that infection occurred at the midpoint between the last negative and first positive tests, a conservative 
estimate since testing often occurs in the context of risk, this would suggest that very few MSM are diagnosed late in infection in King County (e.g. $[15,16])$. However, in an analysis of persons newly diagnosed with HIV in King County from 2005 to 2009, long ITIs did not correspond completely with other measures of late diagnosis such as AIDS diagnosis within one year of HIV diagnosis or CD4 count at diagnosis[13]. Inaccurate reporting of testing history, testing in response to risk, and rapid disease progression among some persons may contribute to these discrepancies. Including ITIs in surveillance efforts may still be useful for assessing efforts to increase testing and for providing a more complete picture of late HIV diagnosis.

\section{Correlates of ITIs}

In our study, being younger, having sex with men only (versus with men and women), being at greater risk for HIV acquisition (according to local criteria), having access to a regular healthcare provider, and seeking testing as part of a regular schedule were associated with increased testing frequency. Similarly, younger MSM were found to have shorter ITIs in a study of STD Clinics in four U.S. cities[7], and younger age[8,9,11], sex with men only[10], having discussed HIV testing with a regular healthcare provider[8], and HIV risk behaviors [8,9] have been associated with testing within the last year in other studies. These studies have also reported associations between testing in the last year and black race[7], Hispanic ethnicity[9], greater income[8,11], and higher levels of education[11], which we did not observe in our study. ITIs may estimate testing frequency more accurately than the proportion of MSM who tested in the last year among individuals approached for participation in research, a strategy which is likely to overestimate frequency. In addition, the metric of proportion testing within the last year is not sufficiently sensitive in a population where testing every three to six months is recommended.

Higher risk MSM may test more often because they adhere to targeted testing recommendations or, if they test in response to perceived exposures, because they engage in behaviors that result in HIV exposure more often than low risk men. More than half of MSM at GCHP reported testing as part of a regular schedule. If the association we observed between testing regularly and shorter ITIs is causal, promoting regular testing could further increase testing frequency. A recent evaluation of National HIV Behavioral Surveillance data suggested that self-reported risk behaviors may not effectively identify those MSM at greatest risk for acquiring HIV and more frequent testing for all sexually active MSM may be warranted[11]. Local data suggest, however, that we can identify those who are at greatest risk[5]. While it is encouraging that men reporting behaviors associated with HIV risk tended to have tested more recently, those newly diagnosed with HIV continued to have longer ITIs than those testing negative. This difference may represent actual differences in testing frequency, differences in ascertainment of last test date, or differences in the duration of risk between those testing newly HIV-positive and -negative.

Surveillance data in this study included MSM diagnosed at the STD Clinic, GCHP, and many other locations around King County. Longer ITIs among all MSM in surveillance compared to MSM testing newly positive at the STD Clinic and GCHP suggests that MSM diagnosed outside of these two large, publicly-funded programs may spend more time unaware of their infection. Publicly-funded HIV programs may be more effective at promoting testing according to public health guidelines than other providers, and public health departments may need to increase outreach in order to improve early identification of HIV among all MSM in their jurisdictions. 


\section{Limitations}

Our study does have limitations. First, single ITIs may not be representative of individuals' typical frequency of testing over time, and ITIs may not accurately reflect time spent HIVinfected but undiagnosed due to variation in reasons for HIV testing. Many ITIs relied on self-reported date of last HIV-negative test and, for surveillance data, date of first positive test or HIV diagnosis and are therefore sensitive to both recall bias and social desirability bias. Finally, STD Clinic and GCHP data include only MSM seeking testing at these sites and may not be representative of all MSM in Seattle.

\section{Conclusions}

In order to reduce the time that HIV-infected persons are unaware of their status, many public health organizations recommend annual testing for all sexually active MSM and more frequent testing for those at greater risk of acquiring HIV[e.g. 3-6,17-19]. However, these recommendations can be vague or inconsistent across jurisdictions, and whether stratifying recommendations by self-reported behavioral risk factors or recommending more frequent testing for all MSM is more effective will depend on the ability to identify those at greatest risk[5,11]. Further efforts are needed to identify, implement, and evaluate testing recommendations for MSM in order to increase opportunities for early identification of HIV infection and linkage to care.

\section{Acknowledgments}

FUNDING

This work was supported by the U.S. National Institute of Mental Health grant number R01 MH086360.

\section{References}

1. Marks G, Crepaz N, Senterfitt JW, et al. Meta-Analysis of High-Risk Sexual Behavior in Persons Aware and Unaware They are Infected with HIV in the United States: Implications for HIV Prevention Programs. J Acquir Immune Defic Syndr. 2005; 39:446-53. [PubMed: 16010168]

2. Cohen MS, Chen YQ, McCauley M, et al. Prevention of HIV-1 Infection with Early Antiretroviral Therapy. N Engl J Med. 2011; 365:493-505. [PubMed: 21767103]

3. Branson BM, Handsfield HH, Lampe MA, et al. Revised Recommendations for HIV Testing of Adults, Adolescents, and Pregnant Women in Health-Care Settings. MMWR. 2006; 55:1-17. [PubMed: 16988643]

4. Workowski KA, Berman S. Sexually Transmitted Diseases Treatment Guidelines, 2010. MMWR. 2010; 59:1-110. [PubMed: 21160459]

5. Menza TW, Hughes JP, Celum CL, et al. Prediction of HIV Acquisition Among Men Who Have Sex With Men. Sex Transm Dis. 2009; 36:547-55. [PubMed: 19707108]

6. Public Health - Seattle \& King County. [Accessed 30 December 2011] Public Health - Seattle \& King County HIV testing and STD screening recommendations for men who have sex with men (MSM). 2010. Available at: http://www.kingcounty.gov/healthservices/health/communicable/std/ providers/msmstd.aspx

7. Helms DJ, Weinstock HS, Mahle KC, et al. HIV Testing Frequency Among Men Who Have Sex With Men Attending Sexually Transmitted Disease Clinics: Implications for HIV Prevention and Surveillance. J Acquir Immune Defic Syndr. 2009; 50:320-6. [PubMed: 19194309]

8. MacKellar DA, Valleroy LA, Anderson JE, et al. Recent HIV Testing Among Young Men Who Have Sex With Men: Correlates, Contexts, and HIV Seroconversion. Sex Transm Dis. 2006; 33:185-92.

9. Manning SE, Thorpe LE, Ramaswamy C, et al. Estimation of HIV Prevalence, Risk Factors, and Testing Frequency among Sexually Active Men Who Have Sex with Men, Aged 18-64 YearsNew York City, 2002. J Urban Health. 2007; 84:212-25. [PubMed: 17295058] 
10. Jeffries WL. HIV testing among bisexual men in the United States. AIDS Educ Prev. 2010; 22:356-70. [PubMed: 20707695]

11. HIV Testing Among Men Who Have Sex With Men - 21 Cities, United States, 2008. MMWR. 2011; 60:694-9. [PubMed: 21637183]

12. Guy R, Goller JL, Spelman T, et al. Does the frequency of HIV and STI testing among MSM in primary care adhere with Australian guidelines? Sex Transm Infect. 2010; 86:371-6. [PubMed: 20460263]

13. Dombrowski JC, Kent JB, Buskin SE, et al. Population-based metrics for the timing of HIV diagnosis, engagement in HIV care, and virologic suppression. AIDS. 2012; 26:77-86. [PubMed: 22008656]

14. Das M, Chu PL, Santos G, et al. Decreases in Community Viral Load Are Accompanied by Reductions in New HIV Infections in San Francisco. PLoS One. 2010; 5:e11068. [PubMed: 20548786]

15. Schacker T, Collier AC, Hughes J, et al. Clinical and Epidemiologic Features of Primary HIV Infection. Ann Int Med. 1996; 125:257-64. [PubMed: 8678387]

16. Gottlieb GS, Heath L, Nickle DC, et al. HIV-1 Variation before Seroconversion in Men Who Have Sex with Men: Analysis of Acute/Early HIV Infection in the Multicenter AIDS Cohort Study. J Infect Dis. 2008; 197:1011-5. [PubMed: 18419538]

17. World Health Organization. [Accessed 30 December 2011] Guidance on provider-initiated HIV testing and counselling in health facilities. Available at: http://www.who.int/hiv/pub/guidelines/ 9789241595568_en.pdf

18. [Accessed 30 December 2011] British HIV Association, British Association of Sexual Health and HIV, British Infection Society. UK National Guidelines for HIV Testing. 2008. Available at: http://www.bhiva.org/documents/Guidelines/Testing/GlinesHIVTest08.pdf

19. Sexually Transmissible Infections in Gay Men Action Group. [Accessed 30 December 2011] Sexually transmitted infection testing guidelines for men who have sex with men. 2010. Available: http://stigma.net.au/resources/STIGMA_MSM_Testing_Guidelines_2010.pdf 


\section{KEY MESSAGES}

- The median HIV intertest interval among MSM in King County, Washington, was less than 9 months.

- Younger MSM, men who had sex with men only (versus men and women), and men at greater risk for HIV reported testing more frequently.

- The intertest interval may be a useful metric in HIV surveillance for assessing testing frequency and late HIV diagnosis.

- Further efforts are needed to identify, implement, and evaluate testing recommendations for MSM in order to identify HIV infections earlier. 


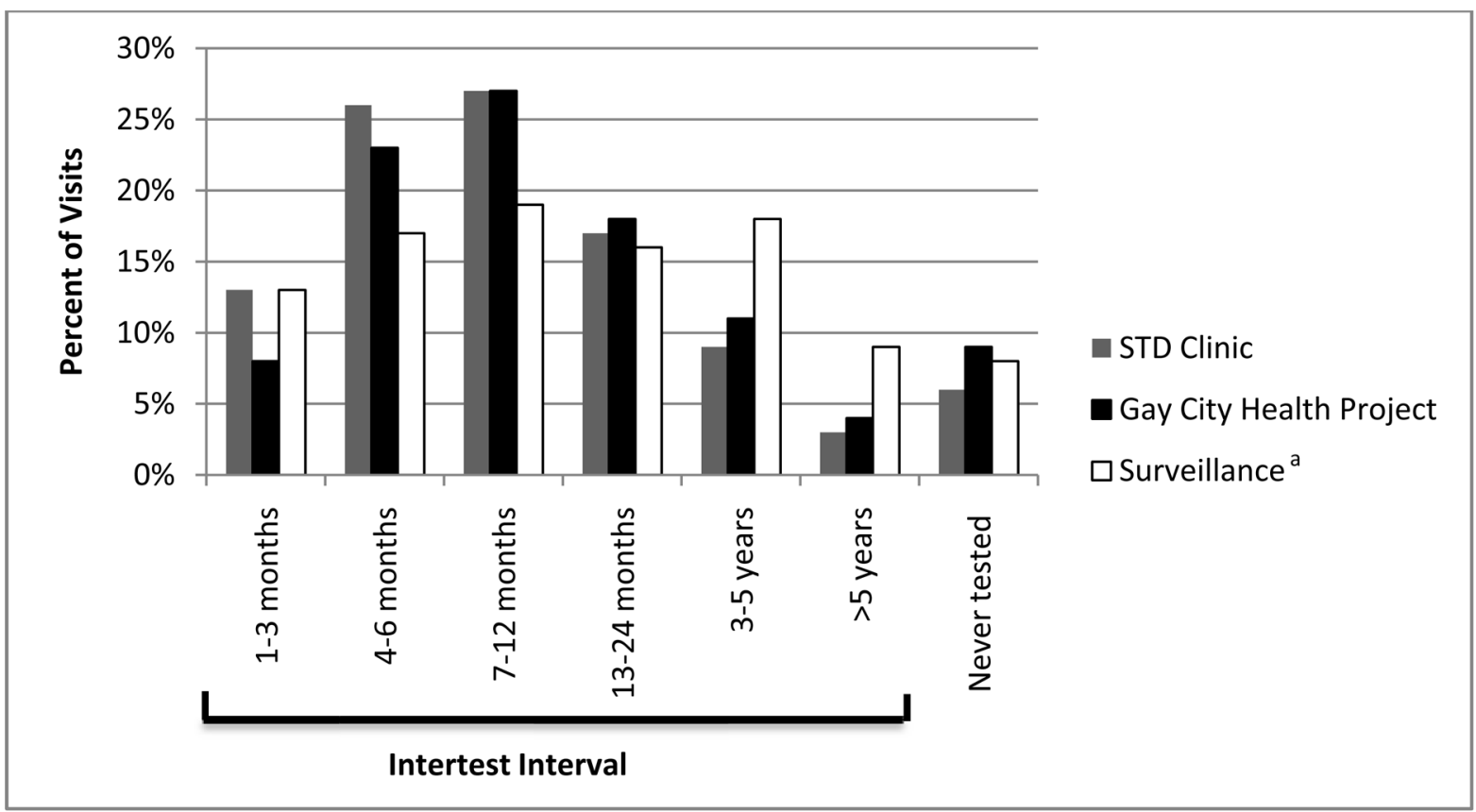

Figure 1. HIV intertest intervals among men who have sex with men in King County, Washington

aData from Public Health - Seattle \& King County (PHSKC) core HIV/AIDS surveillance and HIV incidence surveillance. 
2A: MSM testing newly HIV-positive

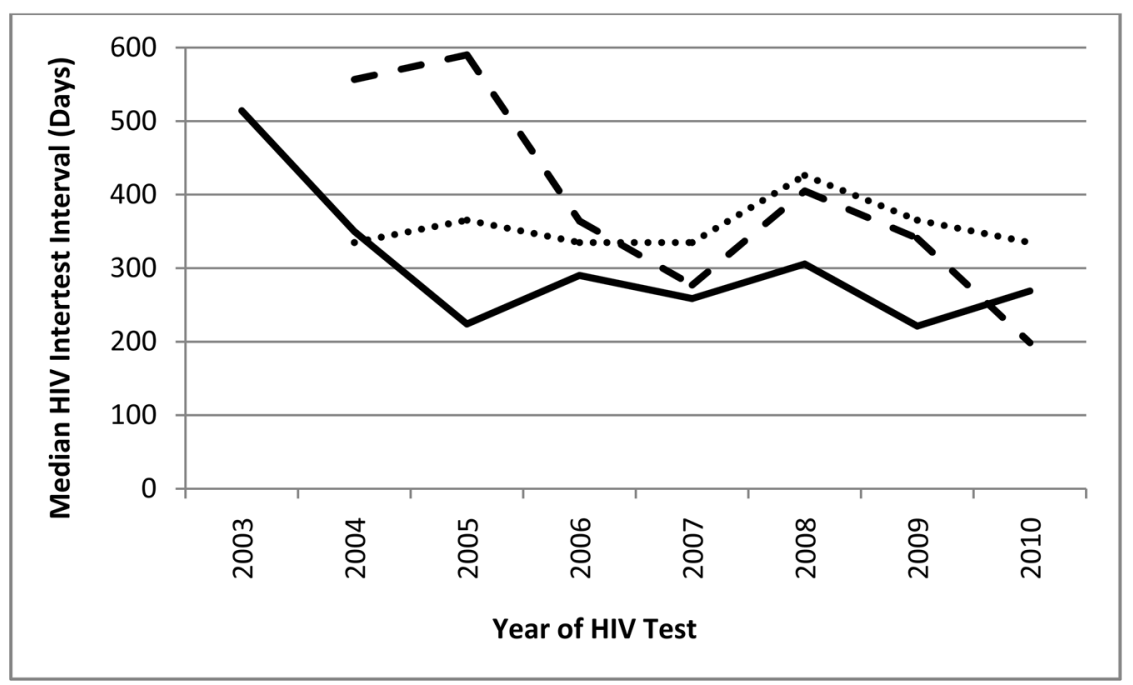

2B: MSM testing newly HIV-negative

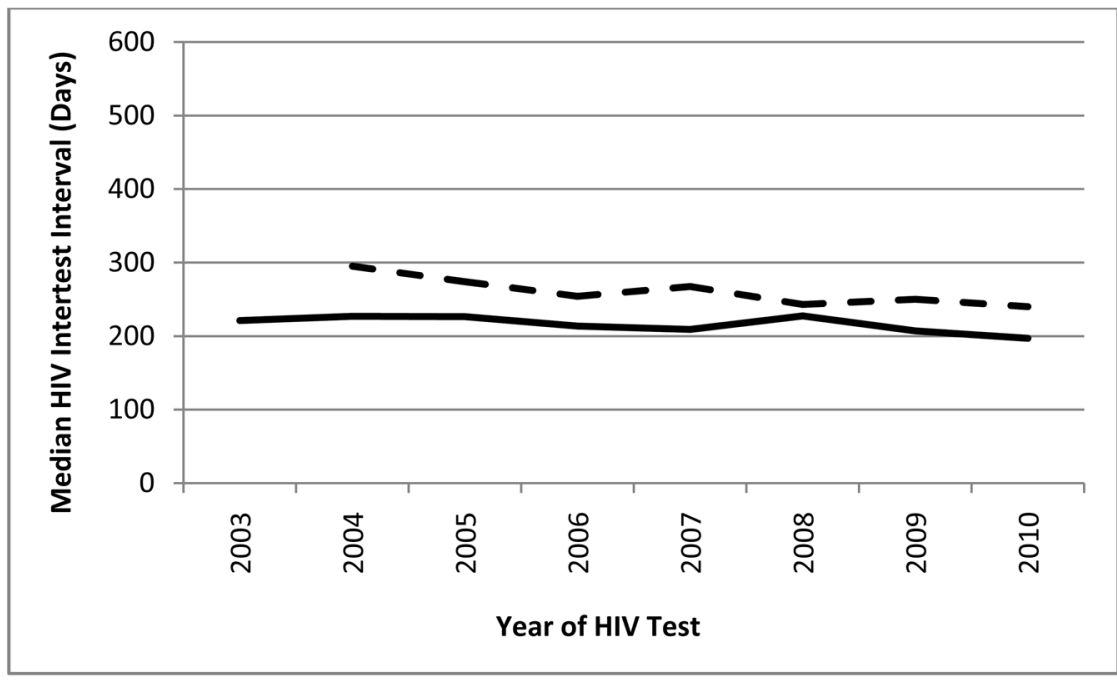

\section{- STD Clinic \\ - - Gay City Health Project \\ Surveillance}

Figure 2.

Temporal trends in HIV intertest intervals among men who have sex with men (MSM) testing newly HIV-positive (A) and HIV-negative (B) in King County, Washington 
Table 1

Characteristics of men who have sex with men seeking HIV testing in publicly-funded programs or newly diagnosed with HIV in King County, Washington

\begin{tabular}{|c|c|c|c|}
\hline \multirow{2}{*}{ Characteristic } & \multicolumn{3}{|c|}{ Median (IQR) or n (\%) } \\
\hline & STD Clinic & Gay City Health Project & Surveillance $^{a}$ \\
\hline $\mathbf{N}$ & 14,385 & 9851 & 1031 \\
\hline Time period & $\begin{array}{l}\text { Jan. 1, } 2003 \text { to Oct. 6, } \\
2010\end{array}$ & $\begin{array}{l}\text { Feb. 2, } 2004 \text { to Oct. 6, } \\
2010\end{array}$ & Jan. 1, 2004 to Oct. 6, 2010 \\
\hline Age (years) & $32(25$ to 41$)$ & $32(25$ to 40$)$ & 35 (27 to 42$)$ \\
\hline \multicolumn{4}{|l|}{$\operatorname{Race}^{b}$} \\
\hline White & $10,619(80 \%)$ & $7089(79 \%)$ & $727(71 \%)$ \\
\hline Black & $979(7 \%)$ & $401(4 \%)$ & $85(8 \%)$ \\
\hline Asian & $955(7 \%)$ & $729(8 \%)$ & $47(5 \%)$ \\
\hline Hawaiian Native/Pacific Islander & $180(1 \%)$ & $97(1 \%)$ & $6(0.6 \%)$ \\
\hline Native American/Alaskan Native & $162(1 \%)$ & $56(0.6 \%)$ & $5(0.5 \%)$ \\
\hline Multiracial & $389(3 \%)$ & $566(6 \%)$ & $35(3 \%)$ \\
\hline Hispanic/Latino $b, c$ & $1511(11 \%)$ & $1225(13 \%)$ & $126(12 \%)$ \\
\hline $\begin{array}{l}\text { Sex with men \& women, last year (versus men } \\
\text { only) }\end{array}$ & $2328(16 \%)$ & $1030(10 \%)$ & $\mathrm{n} / \mathrm{a}$ \\
\hline$\geq 10$ male sex partners, last year & $4505(33 \%)$ & $2423(26 \%)$ & $\mathrm{n} / \mathrm{a}$ \\
\hline Non-concordant UAI, last year & $4148(29 \%)$ & $2022(21 \%)$ & $\mathrm{n} / \mathrm{a}$ \\
\hline History of STI ${ }^{d}$ & $5921(41 \%)$ & $1263(26 \%)$ & $\mathrm{n} / \mathrm{a}$ \\
\hline Methamphetamine use, last year & $1161(8 \%)$ & $544(6 \%)$ & $\mathrm{n} / \mathrm{a}$ \\
\hline Inhaled nitrite use, last year & $2038(14 \%)$ & $1908(19 \%)$ & $\mathrm{n} / \mathrm{a}$ \\
\hline Injection drug use $e^{e}$ & $388(3 \%)$ & $86(0.9 \%)$ & $111(11 \%)$ \\
\hline Newly HIV-diagnosed & $357(2.5 \%)$ & $154(1.6 \%)$ & $1031(100 \%)$ \\
\hline
\end{tabular}

$\mathrm{IQR}=$ interquartile range. $\mathrm{UAI}=$ unprotected anal intercourse. $\mathrm{STI}=$ sexually transmitted infection (syphilis, gonorrhea, or chlamydial infection).

${ }^{a}$ Data from Public Health - Seattle \& King County (PHSKC) core HIV/AIDS surveillance and HIV incidence surveillance.

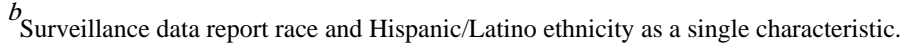

${ }^{c}$ Any race.

${ }^{d}$ At GCHP, history of STI was only collected from 2008 to 2010.

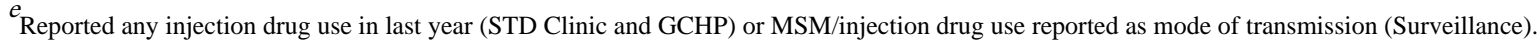


Table 2

Multivariate analyses including sociodemographic and HIV risk characteristics associated with HIV intertest intervals among men who have sex with men, adjusted for year of visit

\begin{tabular}{|c|c|c|c|c|}
\hline \multirow{2}{*}{ Correlates } & \multicolumn{2}{|c|}{ 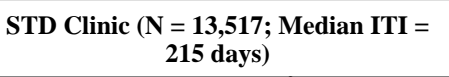 } & \multicolumn{2}{|c|}{$\begin{array}{l}\text { Gay City Health Project }(\mathrm{N}=8995 ; \text { Mediar } \\
\qquad \text { ITI }=257 \text { days })\end{array}$} \\
\hline & $\begin{array}{l}\text { Fold Increase or } \\
\text { Decrease in ITI }\end{array}$ & $95 \% \mathrm{CI}$ & $\begin{array}{l}\text { Fold Increase or } \\
\text { Decrease in ITI }\end{array}$ & $95 \% \mathrm{CI}$ \\
\hline Age (year) & $1.02^{a}$ & 1.01 to 1.02 & $1.02^{a}$ & 1.01 to 1.02 \\
\hline $\begin{array}{l}\text { Sex with men \& women, last year (versus } \\
\text { men only) }\end{array}$ & $1.30^{a}$ & 1.23 to 1.37 & $1.09^{b}$ & 1.01 to 1.19 \\
\hline$\geq 10$ male sex partners, last year & $0.77^{a}$ & 0.75 to 0.80 & $0.78^{a}$ & 0.74 to 0.81 \\
\hline Non-concordant UAI, last year & $0.99^{c}$ & 0.95 to 1.02 & $\mathrm{n} / \mathrm{a}$ & $\mathrm{n} / \mathrm{a}$ \\
\hline History of STI & $0.82^{a}$ & 0.79 to 0.85 & $\mathrm{n} / \mathrm{a}$ & $\mathrm{n} / \mathrm{a}$ \\
\hline Inhaled nitrite use, last year & $0.97^{c}$ & 0.92 to 1.01 & $0.92^{a}$ & 0.87 to 0.97 \\
\hline $\begin{array}{l}\text { Has regular healthcare provider or } \\
\text { doctor }\end{array}$ & $\mathrm{n} / \mathrm{a}$ & $\mathrm{n} / \mathrm{a}$ & $0.84^{a}$ & 0.81 to 0.88 \\
\hline $\begin{array}{l}\text { Reported seeking this test as part of a } \\
\text { regular schedule }\end{array}$ & $\mathrm{n} / \mathrm{a}$ & $\mathrm{n} / \mathrm{a}$ & $0.84^{a}$ & 0.81 to 0.88 \\
\hline Visit year & $0.99^{c}$ & 0.99 to 1.00 & $0.97^{a}$ & 0.96 to 0.98 \\
\hline
\end{tabular}

ITI $=$ intertest interval, in days. $\mathrm{CI}=$ confidence interval. $\mathrm{UAI}=$ unprotected anal intercourse.

STI = sexually transmitted infection; syphilis, gonorrhea, or chlamydial infection.

${ }_{p}^{a}<0.001$.

$b_{p}<0.05$.

${ }_{p}>0.05$. 